\title{
Integral Cross Sections for Electron-Zinc Scattering over a Broad Energy Range (0.01-5000 eV)
}

Cite as: J. Phys. Chem. Ref. Data 49, 013102 (2020); https://doi.org/10.1063/1.5135573

Submitted: 05 November 2019 . Accepted: 10 December 2019 . Published Online: 30 January 2020

R. P. McEachran, B. P. Marinković (D), G. García (D), R. D. White (D), P. W. Stokes (D), D. B. Jones (D), and M. J. Brunger (D)

\section{COLLECTIONS}

F This paper was selected as Featured
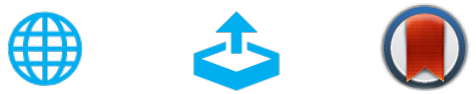

\section{ARTICLES YOU MAY BE INTERESTED IN}

Cross Sections for Electron Collisions with $\mathrm{NO}, \mathrm{N}_{2} \mathrm{O}$, and $\mathrm{NO}_{2}$

Journal of Physical and Chemical Reference Data 48, 043104 (2019); https://

doi.org/10.1063/1.5114722

Wide-Ranging Reference Correlations for Dilute Gas Transport Properties Based on Ab Initio Calculations and Viscosity Ratio Measurements

Journal of Physical and Chemical Reference Data 49, 013101 (2020); https://

doi.org/10.1063/1.5125100

Positron Scattering from Gas-Phase Beryllium and Magnesium: Theory, Recommended Cross Sections, and Transport Simulations

Journal of Physical and Chemical Reference Data 48, 033103 (2019); https://

doi.org/10.1063/1.5115353

Journal of Physical and Chemical Reference Data SPECIAL TOPIC: International Water Property Standards 


\title{
Integral Cross Sections for Electron-Zinc Scattering over a Broad Energy Range (0.01-5000 eV)
}

\author{
Cite as: J. Phys. Chem. Ref. Data 49, 013102 (2020); doi: 10.1063/1.5135573 \\ Submitted: 5 November 2019 • Accepted: 10 December 2019 • \\ Published Online: 30 January 2020
}

\author{
R. P. McEachran, ${ }^{7}$ B. P. Marinković, ${ }^{2}$ (D) Garcia, ${ }^{3}$ (D) R. D. White, ${ }^{4}$ (D) P. W. Stokes, ${ }^{4}$ D. B. Jones, ${ }^{5}$ \\ and M. J. Brunger ${ }^{5,6, a)}$ (iD

\begin{abstract}
AFFILIATIONS
${ }^{1}$ Plasma Research Laboratory, The Research School of Physics, Australian National University, Canberra, A.C.T. 0200, Australia

${ }^{2}$ Institute of Physics Belgrade, University of Belgrade, Pregrevica 118, 11080 Belgrade, Serbia

${ }^{3}$ Instituto de Física Fundamental, CSIC, Serrano 113-bis, E-28006, Madrid, Spain

${ }^{4}$ College of Science and Engineering, James Cook University, Townsville, Queensland 4810, Australia

${ }^{5}$ College of Science and Engineering, Flinders University, GPO Box 2100, Adelaide, S.A. 5001, Australia

${ }^{6}$ Department of Actuarial Science and Applied Statistics, Faculty of Business and Information Science, UCSI University, Kuala Lumpur 56000, Malaysia
\end{abstract}

a) Author to whom correspondence should be addressed: michael.brunger@flinders.edu.au

\begin{abstract}
We report results from the application of our optical potential and relativistic optical potential methods to electron-zinc scattering. The energy range of this study was $0.01-5000 \mathrm{eV}$, with original results for the summed discrete electronic-state integral excitation cross sections and total ionization cross sections being presented here. When combined with our earlier elastic scattering data [Marinković et al., Phys. Rev. A 99, 062702 (2019)], and the quite limited experimental and theoretical results for those processes from other groups, we critically assemble a recommended integral cross section database for electron-zinc scattering. Electron transport coefficients are subsequently calculated for reduced electric fields ranging from 0.1 to $1000 \mathrm{Td}$, using a multiterm solution of Boltzmann's equation. Some differences with corresponding results from the earlier study of White et al. [J. Phys. D: Appl. Phys. 37, 3185 (2004)] were noted, indicating in part the necessity of having accurate and complete cross section data, over a wide energy regime, when undertaking such transport simulations.
\end{abstract}

Published by AIP Publishing on behalf of the National Institute of Standards and Technology. https://doi.org/10.1063/1.5135573

Key words: electron scattering cross sections; electron transport; recommended cross section data; zinc.

\section{CONTENTS}

1. Introduction $\ldots \ldots \ldots \ldots \ldots \ldots \ldots \ldots \ldots$

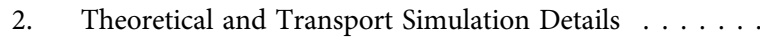

3. Results and Discussion . . . . . . . . . . . .

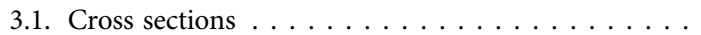

3.2. Transport simulations $\ldots \ldots \ldots \ldots \ldots \ldots$

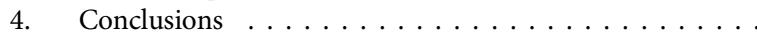
Acknowledgments . . . . . . . . . . . . . . .

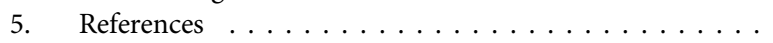

\section{List of Tables}

1. The values we employed in our BEB calculation of the TICS for electron-zinc scattering $\ldots \ldots \ldots \ldots \ldots$
2. A representative selection of recommended cross sections $\left(\times 10^{-16} \mathrm{~cm}^{2}\right)$ for elastic, sum over all discrete inelastic, and TICSs for electron scattering from zinc. Also shown is our recommended momentum transfer cross section $\ldots \ldots \ldots \ldots \ldots \ldots \ldots \ldots \ldots \ldots \ldots \ldots \ldots \ldots$

\section{List of Figures}

(a) Integral elastic, summed discrete inelastic and total ionization cross sections $\left(\times 10^{-16} \mathrm{~cm}^{2}\right)$ for electron scattering from zinc Recommended integral cross sections for elastic (-), sum of discrete inelastic (- - - ), and total ionization (- -) cross sections, as derived as a part of this study, for electron scattering from zinc (see also the legend) $\ldots \ldots \ldots \ldots$ 
3. Comparison between the calculated transport and rate coefficients over a range of reduced electric fields for electron

\section{Introduction}

We have recently been interested in electron ${ }^{1,2}$ and positron scattering from Beryllium (Be) and Magnesium ( $\mathrm{Mg}$ ), both of which represent quasi-two-electron atoms for which a [core]ns ${ }^{2}$ targetconfiguration description has been previously quite successful in describing their scattering phenomena. Zinc ( $\mathrm{Zn}$ ), another quasi-twoelectron atom, represents a natural extension of those earlier investigations, ${ }^{1-3}$ and indeed, we reported new experimental and optical potential (OP) and relativistic optical potential (ROP) calculation results for elastic scattering from that atom earlier in 2019. Here, we extend the original $\mathrm{Zn}$ study to now report OP and ROP integral cross section results for the discrete inelastic (electronicexcitation) channels and the total ionization cross section (TICS). In addition, we examine the presently available results for electron-Zn scattering in order to assemble a recommended set of cross sections for this system. As in our earlier work on $\mathrm{Be}$ and $\mathrm{Mg},{ }^{1-3}$ those recommended integral cross section databases are as complete as we can make them and they are compiled over a broad energy range $(0.01-5000 \mathrm{eV})$. As noted previously by both Tanaka et al. ${ }^{5}$ and the LXCat collaboration, fulfillment of those criteria is essential if charged particle electron transport ${ }^{7}$ or kinetic-radiative modeling simulation results, for zinc vapor, are to be physical.

The latter point is particularly relevant as it has been suggested $^{9,10}$ that $\mathrm{Zn}$ might be an attractive replacement for mercury in making high-pressure discharge lamps more environmentally friendly. Indeed, as a consequence of the work of Born, ${ }^{9,10}$ White et al. ${ }^{7}$ conducted an initial multiterm Boltzmann simulation study looking into the transport characteristics of a swarm of electrons drifting through a background $\mathrm{Zn}$ vapor under the influence of an external electric field $(E)$. However, the energy range of the cross section database in Ref. 7 was quite restricted, which, in turn, limited the range of reduced fields ( $E / n_{0}$, where $n_{0}$ is the neutral density) they could study. As a consequence, we revisit these simulations again here using our recommended cross section database. Recently, ${ }^{8,11}$ studies on the emission dynamics of an expanding ultrafast laser-produced Zn plasma, using a collisional radiative model, have been reported. Additionally, those same authors ${ }^{12}$ have noted that these laserproduced $\mathrm{Zn}$ plasmas also have interesting applications in the generation of higher-order harmonics, attosecond pulses, and in wakefield acceleration. Thus, there are important applied phenomena for which their modeling requires accurate electron-Zn scattering cross sections.

The remainder of this paper is structured as follows: In Sec. 2, we provide very brief details of our OP and ROP calculations. Also in this section, details of our Binary-Encounter-Bethe (BEB) TICS calculations ${ }^{5}$ and our multiterm Boltzmann simulations (e.g., Ref. 2) are given. Our results, including how we formed our recommended cross sections, and a discussion of those results are given in Sec. 3, with some conclusions from the present investigation thereafter being drawn in Sec. 4.

\section{Theoretical and Transport Simulation Details}

As noted above, we have employed two different $a b$ initio theoretical approaches in order to calculate the summed discrete transport in $\mathrm{Zn}$ vapor at $750 \mathrm{~K}$ using the recommended cross section set (Table 2) and the set published by White et al .

inelastic electronic-state integral cross section and the TICS. The first of these is our OP approximation method, which we previously described in some detail in our studies of the electron-beryllium ${ }^{1}$ and electron-magnesium ${ }^{2}$ scattering systems. All the generic details of our atomic OP method, which we gave in those papers, are equally relevant here, and so, as a consequence, we do not repeat them again. Rather, we simply highlight that when our OP method was benchmarked against a quite sophisticated B-spline R-matrix computation, ${ }^{13}$ for electron scattering from atomic iodine, good agreement was found between them, which we hope will carry on in zinc. A similar scenario also applies to our second theoretical method, the ROP approach, which has also been well detailed in some of our earlier work. ${ }^{1,2}$ In this case, however, the particular details pertaining to the zinc computations can be found in Ref. 4 .

In addition, in this investigation, we have also used the BEB formalism ${ }^{5,14}$ to calculate the TICS for electron-zinc scattering. In that approach, the integral ionization cross section $(Q)$, for ionization from atomic orbital $i$, is given by

$$
Q_{i}\left(t_{i}\right)=\frac{4 \pi a_{0}^{2} N_{i}}{t_{i}+\left(u_{i}+1\right) / \eta_{p q n}}\left(\frac{R}{B_{i}}\right)^{2}\left[\frac{\ln t_{i}}{2}\left(1-\frac{1}{t_{i}^{2}}\right)+1-\frac{1}{t_{i}}-\frac{\ln t_{i}}{t_{i}+1}\right] .
$$

In Eq. (1), the binding energy of the ionized orbital, $B_{i}$, is used to scale the incident electron impact energy $\left(E_{0}\right)$ and orbital kinetic energies $\left(U_{i}\right), t_{i}=\frac{E_{0}}{B_{i}}$ and $u_{i}=\frac{U_{i}}{B_{i}}$, respectively. $N_{i}$ is the orbital occupation number, while $R$ and $a_{0}$ are the Rydberg constant and the Bohr radius, respectively. The BEB TICS is then obtained by summing up the contributions from each populated atomic orbital. When applying this method to heavier elements, the scaled kinetic energy is further scaled by the principal quantum number of the ionized orbital $\left(\eta_{p q n}\right)$, if it is greater than 2. In order to apply the BEB model here, we also need to determine a reasonable quantum chemical model for calculating the structural information regarding the $\mathrm{Zn}$ target. Here, we extracted the $\mathrm{Zn}$ structural information from a single point energy calculation of $\mathrm{ZnH}(r=9 \AA)$ that employed a B3LYP/aug-cc-pVTZ model chemistry within the Gaussian $09^{15}$ package. This enables the orbital kinetic energies to be specified. The accuracy of the BEB TICS is very sensitive to the binding energy of the ionized orbital. ${ }^{5} \mathrm{We}$ have, therefore, coupled our calculated orbital kinetic energies with the available experimental binding energies from a photoelectron spectroscopy investigation. ${ }^{16}$ The spectroscopic intensity of the ${ }^{2} \mathrm{D}_{5 / 2}$ and ${ }^{2} \mathrm{D}_{3 / 2}$ features is further used to partition the 10 outer valence (3d) electrons between the accessible final states.

TABLE 1. The values we employed in our BEB calculation of the TICS for electron-zinc scattering

\begin{tabular}{lrrrc}
\hline \hline & $B_{i}(\mathrm{eV})$ & $U_{i}(\mathrm{eV})$ & $N_{i}$ & $\eta_{p q n}$ \\
\hline $4 \mathrm{~s}$ & 9.39 & 35.67 & 2 & 4 \\
$3 \mathrm{~d}_{5 / 2}\left({ }^{2} \mathrm{D}_{5 / 2}\right)$ & 17.17 & 303.42 & 6 & 3 \\
$3 \mathrm{~d}_{3 / 2}\left({ }^{2} \mathrm{D}_{3 / 2}\right)$ & 17.50 & 303.42 & 4 & 3 \\
$3 \mathrm{p}$ & 89.74 & 441.97 & 6 & 3 \\
\hline \hline
\end{tabular}


The veracity of our BEB calculation was assessed by implementing the same procedure for electron scattering from krypton and gallium. Here, we obtained good agreement with available experimental data for krypton, ${ }^{17}$ and we achieved a good reproduction of data from an earlier BEB TICS result for gallium. ${ }^{18}$ We are, therefore, confident in the bona fides of our present implementation of the BEB methodology to Zn, with the parameters we employed in applying Eq. (1) being given in Table 1.

Finally, we note that we calculated transport coefficients for gaseous $\mathrm{Zn}$ at $750 \mathrm{~K}$ using a well benchmarked, multiterm solution of Boltzmann's equation. ${ }^{19,20}$ For comparison, we also applied the twoterm approximation ${ }^{21}$ over the same range of $E / n_{0}$. Here, we found that the results from the multiterm and two-term solvers agreed to better than a few percent for reduced fields less than $100 \mathrm{Td}$. For reduced fields above this, the multiterm transport coefficients can vary by as much as $40 \%$ from the two-term results. This is indicative of a velocity distribution that becomes significantly anisotropic, highlighted by a temperature tensor with elements transverse and parallel to the electric field differing by up to $45 \%$ over the reduced field range considered. There are no qualitative differences in the profiles, however, and the transport coefficients presented later in Sec. 3.2 are those from our two-term solution of the Boltzmann equation.

\section{Results and Discussion}

\subsection{Cross sections}

The currently available integral cross sections for elastic electron scattering from zinc are summarized in Fig. 1(a), which is essentially that given previously in Ref. 4. A full discussion of the results embodied in this figure can also be found in Ref. 4, and so, we do not repeat them again here, except to note three important points. First, there is a strong p-wave resonance at $\sim 0.49 \mathrm{eV}$, which was first observed experimentally by Burrow et al. ${ }^{22}$ Second, there is a higherenergy feature in the elastic ICS at $\sim 5 \mathrm{eV}$, which arises due to the opening of some of the low-lying inelastic channels. Finally, we note that below $1 \mathrm{eV}$ the close-coupling [B-spline R-matrix (BSR) ${ }^{23}$ ] formulation results are always expected to be more reliable than those from the previous OP calculations ${ }^{4}$ and that above $1 \mathrm{eV}$ there is a pretty good level of accord between most of the available theoretical computations ${ }^{4,23-25}$ and with the only experimental ICS results from Ref. 4. This situation makes the formation of a recommended elastic ICS dataset quite straightforward, as now detailed. For energies less than $1 \mathrm{eV}$, we simply used the BSR results, ${ }^{23}$ while for energies above 1 $\mathrm{eV}$ and below $10 \mathrm{eV}$, the ROP data, ${ }^{4}$ scaled by a factor of 0.997187 to ensure continuity with the BSR results, was employed. Between 10 and $100 \mathrm{eV}$, an average of the $\mathrm{ROP},{ }^{4}$ convergent close coupling (CCC), ${ }^{24}$ and $\mathrm{OP}^{4}$ results was taken, and in this case (serendipitously), no scaling was required to ensure continuity with the lower energy ICSs. Finally, for energies between $100 \mathrm{eV}$ and $5000 \mathrm{eV}$, an average of the OP and ROP results ${ }^{4}$ was employed, with the appropriate scaling factor at $100 \mathrm{eV}$ to again ensure continuity now being 0.995354 . A selection of our recommended elastic ICSs for $e^{-}+\mathrm{Zn}$ scattering is given in Table 2, with a graphical representation of that data being found in Fig. 2.

The situation is less clear cut, however, for the sum of the discrete inelastic ICSs. In this case, the available data are restricted to the results from our present ROP and OP computations, an earlier R-
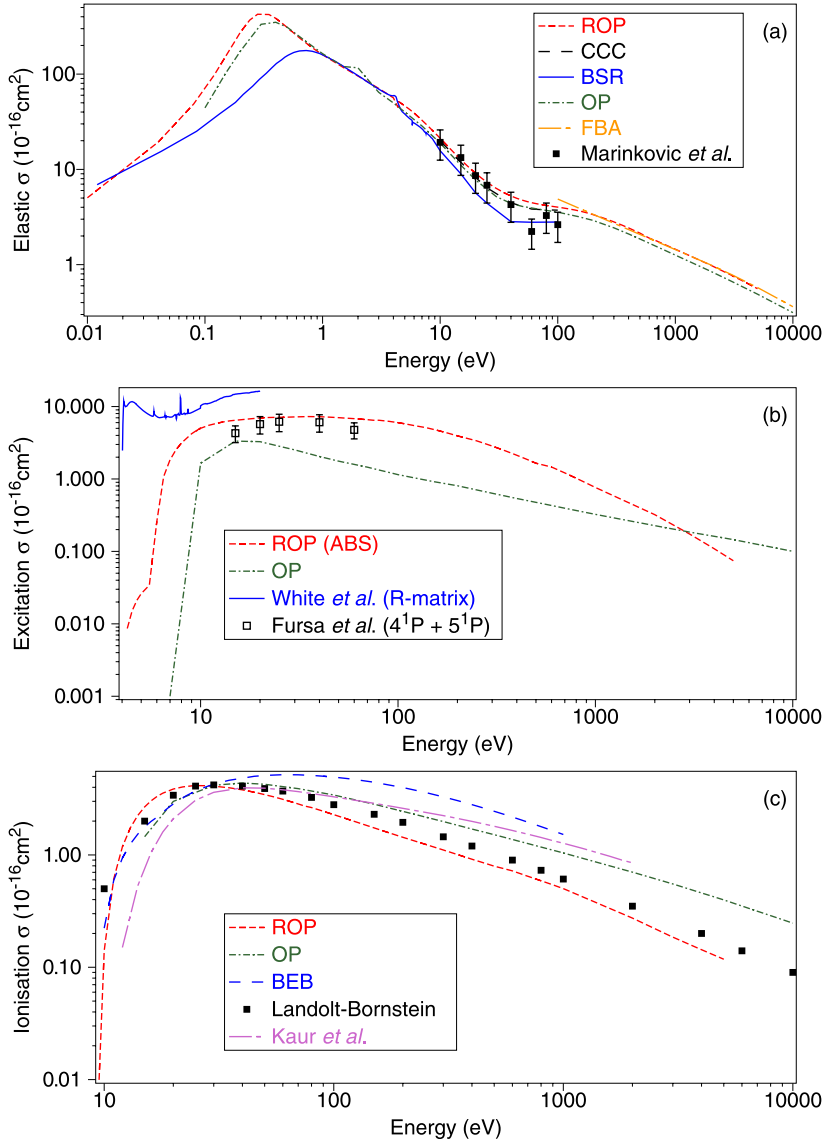

FIG. 1. (a) Integral elastic cross sections $\left(\times 10^{-16} \mathrm{~cm}^{2}\right)$ for electron scattering from zinc. The measurements of Marinkovic et al. ${ }^{4}(\square)$ and their $\mathrm{OP}(-\cdot-\cdot-)$ and $\mathrm{ROP}\left(--\right.$ - $^{-}$calculations are compared against earlier $\operatorname{CCC}^{24}(--), \operatorname{BSR}^{23}(-)$, and $\mathrm{FBA}^{25}(---)$ theoretical results. (b) Integral discrete excitation cross sections $\left(\times 10^{-16} \mathrm{~cm}^{2}\right)$ for electron scattering from zinc: the cross section sum of all inelastic channels is shown for the present ROP $(-\cdot--)$, present $\mathrm{OP}(-\cdot-\cdot-)$, and R-matrix $(-)$ calculations and for the sum of the experimental $4^{1} P+5^{1} P$ integral cross sections ( $\square$ ) from Fursa et al. ${ }^{24}$ (c) TICSs $\left(\times 10^{-16} \mathrm{~cm}^{2}\right)$ for electron scattering from zinc: present ROP (----), present OP $(-\cdot-\cdot-)$, present BEB $(--)$, Landolt-Bornstein ${ }^{26}(\mathbf{\square})$, and Kaur et al..$^{27}(--)$ (see also legends).

matrix result from White et al., ${ }^{7}$ and a sum of the measured optically allowed $4^{1} \mathrm{P}$ and $5^{1} \mathrm{P}$ ICSs from Fursa et al. ${ }^{24}$ All these available data are plotted in Fig. 1(b), where the level of agreement between them is seen to be quite marginal. The only exception to this is between our current ROP calculation and the $4^{1} \mathrm{P}+5^{1} \mathrm{P}$ experimental ICSs from Fursa et al. ${ }^{24}$ However, that agreement must be fortuitous as the ROP computation includes all the integral cross sections for the open discrete inelastic channels at a given incident electron energy, while the Fursa et al. experimental sum only includes a small (but important) subset of those channels (i.e., two). Nonetheless, it is very encouraging that the shape (i.e., the qualitative energy dependence) of the present ROP and Fursa et al. ${ }^{24}$ ICSs, in the energy range of overlap, is in very good accord. As a consequence, our recommended ICSs for the sum over all the discrete inelastic channels is found from the Rmatrix result of White et al., ${ }^{7}$ from threshold up to $20 \mathrm{eV}$, and for 20 
TABLE 2. A representative selection of recommended cross sections $\left(\times 10^{-16} \mathrm{~cm}^{2}\right)$ for elastic, sum over all discrete inelastic, and TICSs for electron scattering from zinc. Also shown is our recommended momentum transfer cross section

\begin{tabular}{|c|c|c|c|c|}
\hline Energy $(\mathrm{eV})$ & Elastic $\left(\times 10^{-16} \mathrm{~cm}^{2}\right)$ & Inelastic $\left(\times 10^{-16} \mathrm{~cm}^{2}\right)$ & $\operatorname{TICS}\left(\times 10^{-16} \mathrm{~cm}^{2}\right)$ & $\operatorname{MTCS}\left(\times 10^{-16} \mathrm{~cm}^{2}\right)$ \\
\hline $0.121 \times 10^{-1}$ & 6.91 & & & 10.2 \\
\hline $0.160 \times 10^{-1}$ & 8.02 & & & 11.9 \\
\hline $0.253 \times 10^{-1}$ & 10.6 & & & 16.0 \\
\hline $0.423 \times 10^{-1}$ & 15.4 & & & 23.4 \\
\hline $0.801 \times 10^{-1}$ & 24.0 & & & 35.1 \\
\hline 0.120 & 34.6 & & & 46.8 \\
\hline 0.181 & 50.3 & & & 59.8 \\
\hline 0.290 & 88.2 & & & 87.8 \\
\hline 0.405 & 129 & & & 111 \\
\hline 0.569 & 169 & & & 127 \\
\hline 0.999 & 159 & & & 99.2 \\
\hline 1.50 & 117 & & & 65.5 \\
\hline 2.00 & 95.4 & & & 49.7 \\
\hline 3.00 & 70.6 & & & 32.3 \\
\hline 4.02 & 55.6 & 2.47 & & 22.3 \\
\hline 4.08 & 53.7 & 11.9 & & 21.2 \\
\hline 4.12 & 52.5 & 10.5 & & 20.5 \\
\hline 4.38 & 44.6 & 11.4 & & 16.1 \\
\hline 4.41 & 51.1 & 11.5 & & 19.4 \\
\hline 5.68 & 41.2 & 7.36 & & 13.4 \\
\hline 5.78 & 40.5 & 7.65 & & 13.0 \\
\hline 5.80 & 40.3 & 8.63 & & 12.9 \\
\hline 5.82 & 40.2 & 8.05 & & 12.9 \\
\hline 6.16 & 37.9 & 7.04 & & 11.6 \\
\hline 6.60 & 35.0 & 7.64 & & 9.99 \\
\hline 6.66 & 34.7 & 7.00 & & 9.81 \\
\hline 7.44 & 30.5 & 7.76 & & 7.71 \\
\hline 7.54 & 30.0 & 6.75 & & 7.49 \\
\hline 7.58 & 29.8 & 7.73 & & 7.41 \\
\hline 7.88 & 28.5 & 8.12 & & 6.82 \\
\hline 7.90 & 28.4 & 13.3 & & 6.78 \\
\hline 7.98 & 28.0 & 8.16 & & 6.63 \\
\hline 8.60 & 25.6 & 8.00 & & 5.64 \\
\hline 8.64 & 25.4 & 9.30 & & 5.59 \\
\hline 8.66 & 25.3 & 8.15 & & 5.56 \\
\hline 9.50 & 22.6 & 8.88 & $0.995 \times 10^{-2}$ & 4.56 \\
\hline 10.0 & 21.1 & 10.2 & 0.141 & 4.08 \\
\hline 11.0 & 18.1 & 11.0 & 0.617 & 3.20 \\
\hline 15.0 & 11.9 & 14.9 & 2.26 & 1.68 \\
\hline 20.0 & 8.37 & 16.3 & 3.41 & 1.06 \\
\hline 26.0 & 6.54 & 16.7 & 4.04 & 0.833 \\
\hline 40.0 & 4.70 & 17.0 & 4.37 & 0.711 \\
\hline 60.0 & 4.08 & 16.0 & 4.22 & 0.814 \\
\hline 99.9 & 3.77 & 14.1 & 3.66 & 1.02 \\
\hline 140 & 3.49 & 11.9 & 3.23 & 1.06 \\
\hline 200 & 3.12 & 9.25 & 2.75 & 0.987 \\
\hline 300 & 2.61 & 6.69 & 2.23 & 0.799 \\
\hline 500 & 1.99 & 3.88 & 1.65 & 0.528 \\
\hline 800 & 1.54 & 2.40 & 1.21 & 0.328 \\
\hline 1000 & 1.35 & 1.79 & 1.03 & 0.253 \\
\hline 1810 & 0.998 & 0.953 & 0.650 & 0.135 \\
\hline 2000 & 0.915 & 0.755 & 0.561 & 0.107 \\
\hline
\end{tabular}


TABLE 2. (Continued.)

\begin{tabular}{lcccc}
\hline \hline Energy $(\mathrm{eV})$ & Elastic $\left(\times 10^{-16} \mathrm{~cm}^{2}\right)$ & Inelastic $\left(\times 10^{-16} \mathrm{~cm}^{2}\right)$ & TICS $\left(\times 10^{-16} \mathrm{~cm}^{2}\right)$ & MTCS $\left(\times 10^{-16} \mathrm{~cm}^{2}\right)$ \\
\hline 3000 & 0.718 & 0.408 & 0.379 & $0.607 \times 10^{-1}$ \\
4000 & 0.610 & 0.255 & 0.294 & $0.409 \times 10^{-1}$ \\
5000 & 0.522 & 0.174 & 0.241 & $0.292 \times 10^{-1}$ \\
\hline \hline
\end{tabular}

$\mathrm{eV}-5000 \mathrm{eV}$ from our ROP result that is scaled upward by a factor of 2.34739 to ensure continuity with the lower energy result. A summary of this recommended ICS data can also be found in Table 2, with its corresponding plot being given in Fig. 2. It is also interesting to note, and possibly of importance in charged-particle transport simulations, that the above scaling factor and our ROP summed cross section can, in principle, be employed to extend the energy range of each of the individual discrete electronic-state excitation cross sections of White

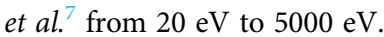

An earlier recommended TICS for electron scattering from $\mathrm{Zn}$ was previously compiled, from the then available experimental and theoretical data, by Inokuti. ${ }^{26}$ Those TICS are plotted in Fig. 1(c) along with our present OP and ROP computational results, a BEB model TICS we computed as a part of this study, and a relatively recent calculation from Kaur and colleagues. ${ }^{27}$ Tanaka et al. ${ }^{5}$ examined in some detail the efficacy of the BEB approach and found that in many cases its results agreed well with reliable TICS data, where available, to better than $25 \%$. As a consequence, and even though our ROP result is actually in pretty good agreement with the earlier recommended TICS set from Inokuti ${ }^{26}$ [see Fig. 1(c)] over their common energy range, our recommended TICS database is now formed as follows. For energies from threshold to $11 \mathrm{eV}$, we adopt our ROP result, which from Fig. 1(c) is clearly trending to the correct first

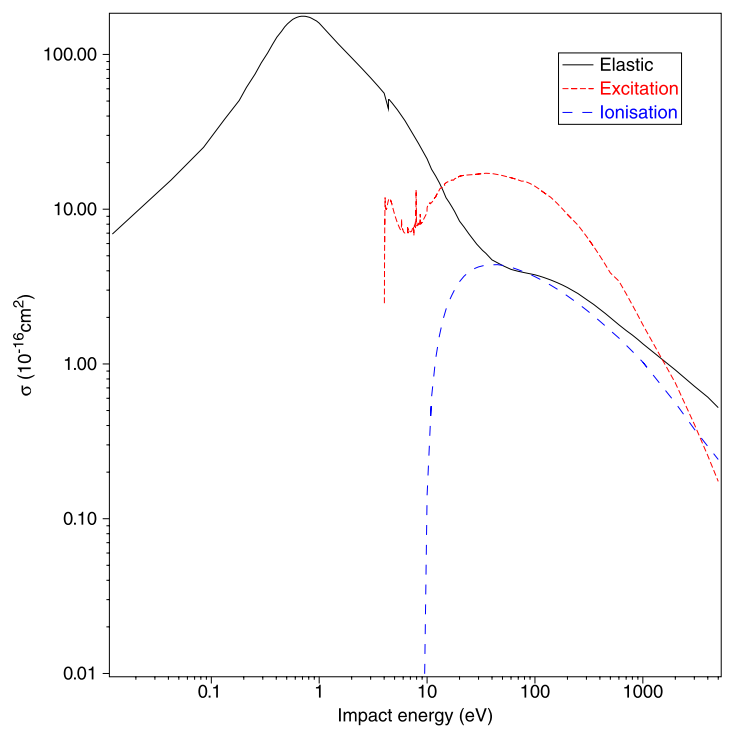

FIG. 2. Recommended integral cross sections for elastic (-), sum of discrete inelastic (----), and total ionization (--) cross sections, as derived as a part of this study, for electron scattering from zinc (see also the legend). ionization energy of zinc. For energies in the range $11 \mathrm{eV}-1000 \mathrm{eV}$, we adopt an average of the ROP and BEB TICS, scaled by a factor of 1.01547 to ensure continuity at $11 \mathrm{eV}$ with the lower energy data. Finally, for energies in the range $1000 \mathrm{eV}-5000 \mathrm{eV}$, we again simply choose our ROP TICS, now scaled upward by a factor of 2.04746 for the purpose of continuity, to complete our recommended TICS. The resultant TICS is tabulated in Table 2 and plotted in Fig. 2. Note that although we do not explicitly show it, our recommended TICS is found to be in quite good agreement with that compiled earlier by Inokuti, ${ }^{26}$ thereby indicating what a thorough job that author did with the data available to him.

Finally, for both the sake of completeness and because it is important when attempting to account for anisotropic scattering in our transport simulations, we note that a recommended momentum transfer cross section (MTCS) was also determined as a part of this investigation. This MTCS was formed in the same manner as in our earlier work on Be and $\mathrm{Mg} .{ }^{1-3}$ Specifically, the recommended MTCS is given by the recommended elastic ICS scaled by the ratio of our ROP calculated MTCS divided by our ROP elastic ICS. A selection of the present recommended MTCS can be found in Table 2.

\subsection{Transport simulations}

Figure 3 plots transport and rate coefficients for an electron swarm in $\mathrm{Zn}$ vapor at $750 \mathrm{~K}$, a temperature that was chosen to be consistent with that used in our earlier $\mathrm{Mg}$ and Be simulations, ${ }^{2,3}$ for the recommended cross section set (Table 2 ) and the set published by White et al. ${ }^{7}$ Over the range of reduced fields $E / n_{0}$ considered from $10^{-3} \mathrm{Td}$ to $10^{3} \mathrm{Td}$, we compare the bulk drift velocity $W$, the bulk longitudinal diffusion coefficient $n_{0} D_{L}$, the mean energy $\varepsilon$, and the ionization rate coefficient $k_{\text {io }}$ between the two sets. We reiterate that the two-term approximation was found to be sufficient to within a few percent over the range of $E / n_{0}$ up to $100 \mathrm{Td}$. The two-term approximation did break down for reduced fields above this with transport coefficients differing by up to $40 \%$ at the maximum field considered. ${ }^{19,20}$ We observe an almost order of magnitude lower bulk drift and diffusion for the recommended cross section set at very low $E / n_{0}\left(\sim 10^{-3} \mathrm{Td}\right)$. This is entirely due to the higher magnitude elastic MTCS for the recommended set compared to the White et al. ${ }^{7}$ set in the thermal energy regime $\left(\sim 10^{-1} \mathrm{Td}\right)$. Conversely, at high $E / n_{0}$, transport coefficients for the White et al..$^{7}$ set become smaller relative to those of the recommended set due to a corresponding relative increase in the magnitude of the White et al. ${ }^{7}$ elastic MTCS at high energies. This effect is also reflected by the mean energy, which is larger for the White et al. ${ }^{7}$ set for much of the considered range of $E / n_{0}$, only dipping below that for the recommended set beyond $\sim 425 \mathrm{Td}$. The ionization rates are almost equivalent for both sets due to the similar ionization and summed excitation channel integral cross sections in each one. The ionization rate does have a slightly lower 

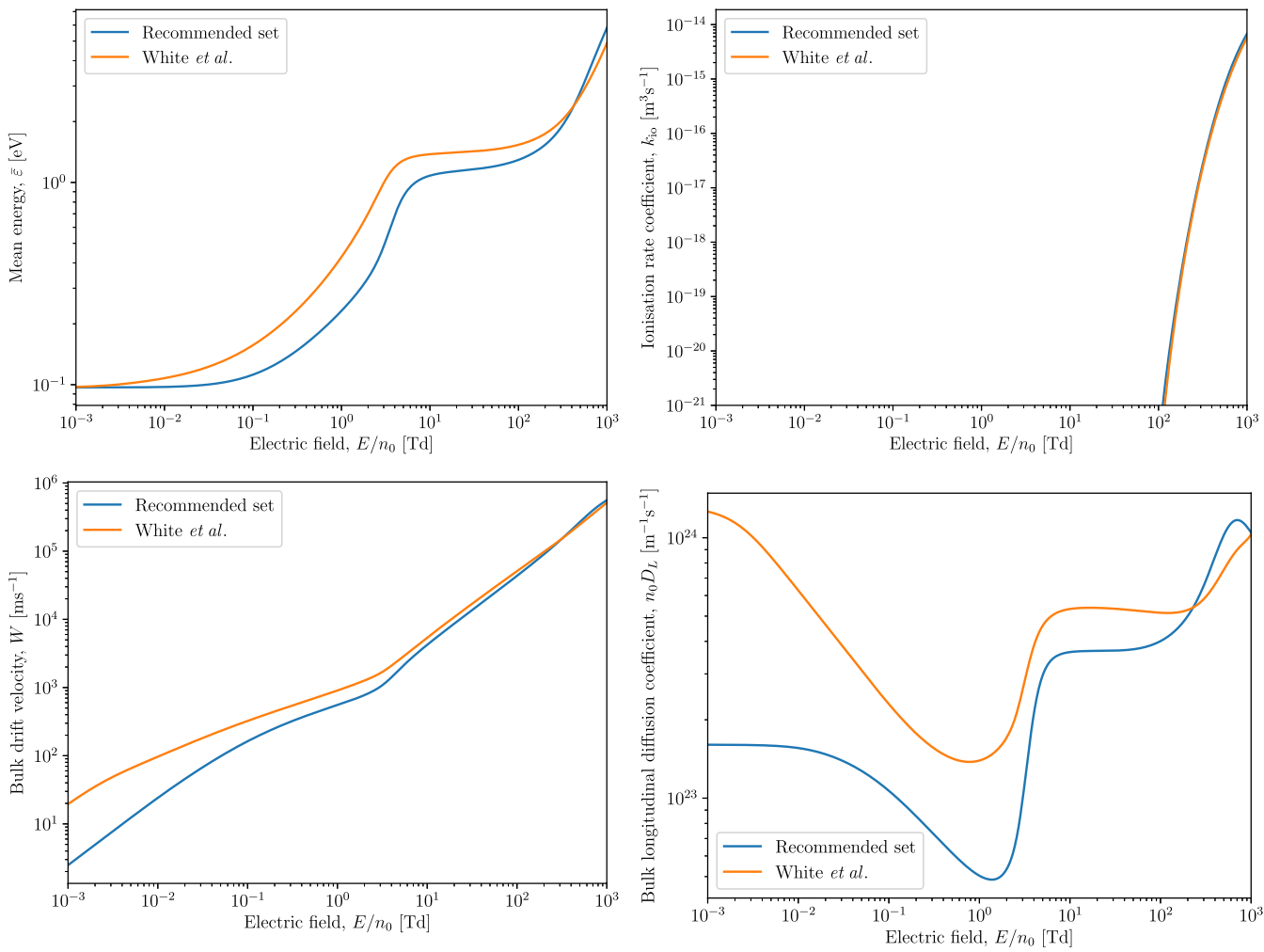

FIG. 3. Comparison between the calculated transport and rate coefficients over a range of reduced electric fields for electron transport in $\mathrm{Zn}$ vapor at $750 \mathrm{~K}$ using the recommended cross section set (Table 2) and the set published by White et al. ${ }^{7}$

threshold $E / n_{0}$ for the recommended set due to its smaller ionization threshold energy of $\sim 9.5 \mathrm{eV}$ vs $\sim 10 \mathrm{eV}$ for the ionization cross section used by White et al.

Tables of the present swarm simulation results, for both the twoterm and multiterm applications, can be obtained by contacting coauthor White. ${ }^{28}$

\section{Conclusions}

We have critically examined, in detail, the available integral cross section data for electron scattering from zinc, and from that process compiled recommended cross sections for elastic scattering, the sum of all discrete excited electronic-states and the TICS. In addition, a recommended MTCS was also determined. Subsequently, all these recommended data were employed in a Boltzmann equation analysis in order to study the transport of electrons under the influence of an applied external electric field through zinc vapor at $750 \mathrm{~K}$. Our results for the relevant transport coefficients were then compared against those from the earlier investigation of White et al., ${ }^{7}$ with in some cases significant differences, particularly at lower values of $E / n_{0}$, being found. These discrepancies could be largely ascribed to the different treatments for anisotropic scattering effects in the two studies, as reflected in their rather disparate MTCSs. That latter observation highlights the importance of employing accurate cross sections when undertaking charged-particle transport simulations, such as here, but also in the Monte Carlo simulation work from the Madrid ${ }^{29,30}$ and Belgrade $^{31}$ groups.

\section{Acknowledgments}

This work was financially supported, in part, by the Spanish Ministerio de Economia y Competitividad (Project No. FIS201680440) and the Australian Research Council (Project Nos. DP160102787 and DP180101655). Financial support from the Ministry of Education, Science and Technological Development (Project No. OI171020) of the Republic of Serbia is also acknowledged. We thank Dr. L. Campbell for his help with some aspects of this study.

\section{References}

${ }^{1}$ R. P. McEachran, F. Blanco, G. García, and M. J. Brunger, J. Phys. Chem. Ref. Data 47, 033103 (2018).

${ }^{2}$ R. P. McEachran, F. Blanco, G. García, P. W. Stokes, R. D. White, and M. J. Brunger, J. Phys. Chem. Ref. Data 47, 043104 (2018).

${ }^{3}$ F. Blanco, G. García, R. P. McEachran, P. W. Stokes, R. D. White, and M. J. Brunger, J. Phys. Chem. Ref. Data 48, 033103 (2019).

${ }^{4}$ B. P. Marinković, R. Panajotović, D. Šević, R. P. McEachran, G. García, F. Blanco, and M. J. Brunger, Phys. Rev. A 99, 062702 (2019).

${ }^{5}$ H. Tanaka, M. J. Brunger, L. Campbell, H. Kato, M. Hoshino, and A. R. P. Rau, Rev. Mod. Phys. 88, 025004 (2016). 
${ }^{6}$ L. C. Pitchford, L. L. Alves, K. Bartschat, S. F. Biagi, M.-C. Bordage, I. Bray, C. E. Brion, M. J. Brunger, L. Campbell et al., Plasma Process. Polym. 14, 1600098 (2017). ${ }^{7}$ R. D. White, R. P. McEachran, R. E. Robson, M. T. Elford, and K. Bartschat, J. Phys. D: Appl. Phys. 37, 3185 (2004).

${ }^{8}$ N. Smijesh and R. Philip, J. Appl. Phys. 114, 093301 (2013).

${ }^{9}$ M. Born, J. Phys. D: Appl. Phys. 34, 909 (2001).

${ }^{10}$ M. Born, Plasma Sources Sci. Technol. 11, A55 (2002).

${ }^{11}$ S. Gupta, R. K. Gangwar, and R. Srivastava, in Proceedings of the International Conference on Atomic Processes in Plasmas (NIST, Gaithersburg, MD, USA, 2019).

${ }^{12}$ S. Gupta, R. K. Gangwar, and R. Srivastava, in Abstracts of the 2019 Gaseous Electronics Conference, College Station, TX, USA, 2019.

${ }^{13}$ O. Zatsarinny, K. Bartschat, G. Garciá, F. Blanco, L. R. Hargreaves, D. B. Jones, R. Murrie, J. R. Brunton, M. J. Brunger, M. Hoshino, and S. J. Buckman, Phys. Rev. A 83, 042702 (2011)

${ }^{14}$ Y.-K. Kim and M. E. Rudd, Phys. Rev. A 50, 3954 (1994).

${ }^{15} \mathrm{M}$. J. Frisch et al., GAUSSIAN 09, Revision B.01, Gaussian, Inc., Wallingford, CT, 2010.

${ }^{16}$ S. Süzer, S. T. Lee, and D. A. Shirley, Phys. Rev. A 13, 1842 (1976).

${ }^{17}$ A. A. Sorokin, L. A. Shmaenok, S. V. Bobashev, B. Möbus, M. Richter, and G. Ulm, Phys. Rev. A 61, 022723 (2000).

${ }^{18}$ Y.-K. Kim and P. M. Stone, Phys. Rev. A 64, 052707 (2001).

${ }^{19}$ G. J. Boyle, W. J. Tattersall, D. G. Cocks, R. P. McEachran, and R. D. White, Plasma Sources Sci. Technol. 26, 024007 (2017).

${ }^{20}$ R. D. White, D. Cocks, G. Boyle, M. Casey, N. Garland, D. Konovalov, B. Philippa, P. Stokes, J. de Urquijo, O. González-Magaña, R. P. McEachran, S. J. Buckman, M. J.
Brunger, G. Garcia, S. Dujko, and Z. Lj. Petrovic, Plasma Sources Sci. Technol. 27, 053001 (2018).

${ }^{21}$ R. D. White, R. E. Robson, B. Schmidt, and M. A. Morrison, J. Phys. D: Appl. Phys. 36, 3125 (2003).

${ }^{22}$ P. D. Burrow, J. A. Michejda, and J. Comer, J. Phys. B: At., Mol. Phys. 9, 3225 (1976).

${ }^{23}$ O. Zatsarinny and K. Bartschat, Phys. Rev. A 71, 022716 (2005).

${ }^{24}$ D. V. Fursa, I. Bray, R. Panajotović, D. Šević, V. Pejčev, D. M. Filipović, and B. P. Marinković, Phys. Rev. A 72, 012706 (2005).

${ }^{25}$ S. T. Perkins, D. E. Cullen, and S. M. Seltzer, LLNL Evaluated Electron Data Library, UCRL-50400, Vol. 31, 1991.

${ }^{26} \mathrm{M}$. Inokuti, "Electron collisions with atoms," in Landolt-Börnstein: Group I Elementary Particles, Nuclei and Atoms Vol. 17A, edited by Y. Itikawa (SpringerVerlag, Berlin, 2000).

${ }^{27}$ J. Kaur, D. Gupta, R. Naghma, D. Ghoshal, and B. Antony, Can. J. Phys. 93, 617 (2015).

${ }^{28}$ See ronald.white@jcu.edu.au for tables of the transport coefficients.

${ }^{29} \mathrm{M}$. J. Brunger, K. Ratnavelu, S. J. Buckman, D. B. Jones, A. Muñoz, F. Blanco, and G. García, Eur. Phys. J. D 70, 46 (2016).

${ }^{30}$ A. I. Lozano, J. C. Oller, D. B. Jones, R. F. da Costa, M. T. do N. Varella, M. H. F. Bettega, F. Ferreira da Silva, P. Limão-Vieira, M. A. P. Lima, R. D. White, M. J. Brunger, F. Blanco, A. Muñoz, and G. García, Phys. Chem. Chem. Phys. 20, 022368 (2018).

${ }^{31}$ Z. Lj. Petrović, S. Marjanović, S. Dujko, A. Banković, G. Malović, S. Buckman, G. García, R. White, and M. Brunger, Appl. Radiat. Isot. 83, 148 (2014). 